\title{
BIOPRODUÇÃO DE CAROTENOIDES POR Xanthophyllomyces dendrorhous Y-10921 EM FRASCOS AGITADOS VARIANDO COMPOSIÇÃO DO MEIO DE CULTURA E CONDIÇÕES OPERACIONAIS
}

\author{
L. URNAU $^{(1)}$, L. TIGGEMANN ${ }^{(2)}$, R. COLET, C. STEFFENS ${ }^{(1)}$, E. VALDUGA $^{(1)}$ \\ ${ }^{(1)}$ Universidade Regional Integrada do Alto Uruguai e das Missões - Campus Erechim, Departamento \\ de Engenharia de Alimentos; \\ (2) Universidade Federal de Santa Catarina, Departamento de Engenharia Química e de Alimentos; \\ E-mail: letiurnau@gmail.com
}

RESUMO- Os carotenoides são corantes naturais que podem ser sintetizados por plantas, algas e micro-organismos. Devido às suas propriedades corantes, ao seu potencial efeito benéfico na saúde humana e ao apelo pela substituição dos corantes sintéticos pelos naturais, os carotenoides têm recebido atenção especial, sendo que sua produção por micro-organismos pode tornar-se industrialmente praticável. Este estudo teve como objetivo analisar a influência do meio de cultura e as condições operacionais da bioprodução, na recuperação de carotenoides específicos produzidos por Xanthophyllmomyces dendrorhous Y-10921 em frascos agitados. A bioprodução foi realizada em frascos agitados em shaker, sem iluminação, variando-se a composição do meio YM (concentração de glicose, peptona, extrato de malte e de levedura), temperatura, agitação e pH inicial. A recuperação dos carotenoides foi realizada empregando dimetilsulfóxido (DMSO) para ruptura celular e extração acetona. A determinação dos carotenoides totais foi realizada espectrofotometricamente $(\lambda=474 \mathrm{~nm})$. A máxima bioprodução de carotenoides $(697 \mu \mathrm{g} / \mathrm{g})$ foi observada no ensaio 1 onde o meio de cultura foi composto apenas por glicose $(10 \mathrm{~g} / \mathrm{L})$, e maiores faixas de temperatura e $\mathrm{pH}\left(30^{\circ} \mathrm{C}\right.$ e 6 , respectivamente).

Palavras-chave: carotenoides, meio de cultura, Xanthophyllmomyces dendrorhous.

\section{INTRODUÇÃO}

Carotenoides são pigmentos lipossolúveis encontrados em fungos, bactérias, tecidos de plantas verdes e também em animais (MEZZOMO, 2012). Apresentam capacidade corante, que provem da presença de uma longa série de duplas ligações conjugadas, que é também responsável por suas propriedades antioxidantes (MACÍAS-SANCHEZ et al. 2010).

Os carotenoides são corantes naturais responsáveis pelas cores vermelha, amarela e laranja, muito empregadas nas indústrias alimentícias, farmacêutica, de cosméticos e até mesmo de ração animal (RODRIGUES-AMAYA, KIMURA, AMAYA-FARFAN, 2008).

A produção biotecnológica de carotenoides para a aplicação industrial, a partir de microorganismos, tem sido item de destaque nos últimos anos, pois a grande maioria dos carotenoides 
utilizados industrialmente é obtida por via química ou por extração de plantas ou algas. Devido à preocupação com o uso de aditivos químicos nos alimentos, os processos biotecnológicos para obtenção de carotenoides têm sido alvo de um crescente interesse (JOHNSON; SCHROEDER, 1995; AKSU; EREN, 2007).

A produção de carotenoides através de via microbiana constitui atualmente uma alternativa interessante devido à possibilidade da obtenção de pigmentos de origem natural em escala industrial (DA SILVA, 2004). Há uma grande quantidade de micro-organismos com capacidade para produzir carotenoides, no entanto, nem todos são industrialmente interessantes. As leveduras destacam-se pela sua utilização como fonte proteica, capacidade de crescimento em substratos menos dispendiosos e alto teor de açúcar (VALDUGA et al, 2009).

O interesse na produção de astaxantina por fontes naturais vem aumentando significativamente nos últimos anos, devido à possibilidade de atuar como corante e sua capacidade antioxidante biológica potente. É o carotenoide principal encontrado na levedura Xanthophyllomyces dendrorhous, sendo esse micro-organismo adequado para o uso como fonte do pigmento industrial em razão de seu metabolismo heterotrófico, padrão de crescimento relativamente rápido, qualidade nutricional e seguro como aditivo alimentar (MACÍASSANCHEZ et al, 2004).

Dentro deste contexto, o trabalho teve por objetivo bioproduzir carotenoides da levedura Xanthophyllomyces dendrorhous Y 10921 através da variação do meio de cultivo e condições operacionais, a fim de otimizar a produção dos mesmos, obtendo-se assim, uma maior recuperação.

\section{MATERIAL E MÉTODOS}

O micro-organismo utilizado foi a levedura Xanthophyllomyces dendrorhous Y-10921 adquirida da United States Department of Agriculture. A produção foi realizada em frascos agitados $\left(25^{\circ} \mathrm{C}\right.$ e $\left.150 \mathrm{rpm}\right)$ com um volume útil de $100 \mathrm{~mL}$, sem iluminação e com $10 \%$ de inóculo, durante 96 horas, com meio produzido de acordo com planejamento experimental variando a composição a partir do meio YM (3 g/L de extrato de levedura, $3 \mathrm{~g} / \mathrm{L}$ de extrato de malte, $5 \mathrm{~g} / \mathrm{L}$ de peptona e $10 \mathrm{~g} / \mathrm{L}$ de glicose).

Para estudar os efeitos da composição do meio de cultura e das condições na maximização e/ou otimizar a bioprodução foi utilizado metodologia de planejamento de experimentos. Inicialmente, um planejamento do tipo Plackett-Burman (Screening Design) de 12 ensaios com 3 pontos centrais. As variáveis estudadas e seus respectivos níveis encontramse descritos na Tabela 2, tendo sido temperatura, agitação, $\mathrm{pH}$, extrato de levedura, extrato de malte, peptona e glicose. 


\subsection{Recuperação dos carotenoides}

\subsubsection{Preparo das amostras}

Inicialmente, as células foram separadas em centrífuga, a $6000 \mathrm{x} \mathrm{g}$, por $10 \mathrm{~min}$ a $5^{\circ} \mathrm{C}$. As células foram utilizadas foram congeladas em freezer a $-80^{\circ} \mathrm{C}$ por aproximadamente $5 \mathrm{~h}$ e secas em liofilizador por aproximadamente $36 \mathrm{~h}$.

\subsubsection{Ruptura celular}

A célula $(0,05 \mathrm{~g})$ foi adicionada de $2 \mathrm{~mL}$ de DMSO e homogeneizadas em shaker a 150 rpm por 30 minutos. Após a ruptura foi adicionado o solvente (acetona) para extração dos carotenoides. A amostra foi centrifugada e as lavagens com solvente repetidas até branqueamento da célula.

A medida da concentração de carotenoides específicos foi feita em espectrofotômetro a $474 \mathrm{~nm}$. O cálculo para determinação foi realizado através da Equação 1, utilizando o coeficiente de absortividade específica para xantofilas de 1600 (LIU, WU \& HO, 2006).

$$
\frac{\mu g}{g}=\frac{A * V * 10^{6}}{A_{1 \mathrm{~cm}}^{1 \%} * 100 * m_{\text {amostra }}}
$$

onde: $\mathrm{A}=$ absorbância; $\mathrm{V}=$ volume $(\mathrm{mL}) ; m_{\text {amostra }}=$ massa celular seca $(\mathrm{g}) ; A_{1 \mathrm{~cm}}^{1 \%}=$ absortividade específica.

\section{RESULTADOS E DISCUSSÃO}

A Tabela 2 apresenta a matriz do planejamento tipo Plackett-Burman com os valores codificados (reais) das variáveis independentes estudadas e as respostas em carotenoides específicos $(\mu \mathrm{g} / \mathrm{g})$. 
Tabela 2- Matriz do planejamento tipo Plackett \& Burmann (valores reais e codificados) e respostas em carotenoides específicos em rompimento celular realizado com ultrassom.

\begin{tabular}{ccccccccc}
\hline \multirow{2}{*}{ Ensaios } & *Variáveis Independentes & & & & & \multicolumn{2}{c}{ Respostas } \\
\cline { 2 - 8 } & $\boldsymbol{X}_{\boldsymbol{I}}$ & $\boldsymbol{X}_{\mathbf{2}}$ & $\boldsymbol{X}_{\mathbf{3}}$ & $\boldsymbol{X}_{\mathbf{4}}$ & $\boldsymbol{X}_{\mathbf{5}}$ & $\boldsymbol{X}_{\boldsymbol{6}}$ & $\boldsymbol{X}_{\mathbf{7}}$ & $\begin{array}{c}\text { Carotenoides específicos } \\
(\boldsymbol{\mu g} / \mathbf{g})\end{array}$ \\
\hline $\mathbf{1}$ & $1(30)$ & $-1(150)$ & $1(6)$ & $-1(0)$ & $-1(0)$ & $-1(0)$ & $-1(10)$ & 697,24 \\
$\mathbf{2}$ & $1(30)$ & $1(200)$ & $-1(4)$ & $1(10)$ & $-1(0)$ & $-1(0)$ & $-1(10)$ & 366,56 \\
$\mathbf{3}$ & $-1(20)$ & $1(200)$ & $1(6)$ & $-1(0)$ & $1(10)$ & $-1(0)$ & $-1(10)$ & 497,5 \\
$\mathbf{4}$ & $1(30)$ & $-1(150)$ & $1(6)$ & $1(10)$ & $-1(0)$ & $1(10)$ & $-1(10)$ & 353,25 \\
$\mathbf{5}$ & $1(30)$ & $1(200)$ & $-1(4)$ & $1(10)$ & $1(10)$ & $-1(0)$ & $1(30)$ & 260,62 \\
$\mathbf{6}$ & $1(30)$ & $1(200)$ & $1(6)$ & $-1(0)$ & $1(10)$ & $1(10)$ & $-1(10)$ & 394,5 \\
$\mathbf{7}$ & $-1(20)$ & $1(200)$ & $1(6)$ & $1(10)$ & $-1(0)$ & $1(10)$ & $1(30)$ & 372,5 \\
$\mathbf{8}$ & $-1(20)$ & $-1(150)$ & $1(6)$ & $1(10)$ & $1(10)$ & $-1(0)$ & $1(30)$ & 230,25 \\
$\mathbf{9}$ & $-1(20)$ & $-1(150)$ & $-1(4)$ & $1(10)$ & $1(10)$ & $1(10)$ & $-1(10)$ & 268,75 \\
$\mathbf{1 0}$ & $1(30)$ & $-1(150)$ & $-1(4)$ & $-1(0)$ & $1(10)$ & $1(10)$ & $1(30)$ & 329,75 \\
$\mathbf{1 1}$ & $-1(20)$ & $1(200)$ & $-1(4)$ & $-1(0)$ & $-1(0)$ & $1(10)$ & $1(30)$ & 552,5 \\
$\mathbf{1 2}$ & $-1(20)$ & $-1(150)$ & $-1(4)$ & $-1(0)$ & $-1(0)$ & $-1(0)$ & $-1(10)$ & 91,25 \\
$\mathbf{1 3}$ & $0(25)$ & $0(180)$ & $0(5)$ & $0(5)$ & $0(5)$ & $0(5)$ & $0(20)$ & 432,5 \\
$\mathbf{1 4}$ & $0(25)$ & $0(180)$ & $0(5)$ & $0(5)$ & $0(5)$ & $0(5)$ & $0(20)$ & 410,25 \\
$\mathbf{1 5}$ & $0(25)$ & $0(180)$ & $0(5)$ & $0(5)$ & $0(5)$ & $0(5)$ & $0(20)$ & 398,75 \\
\hline
\end{tabular}

*X1=Temperatura $\left({ }^{\circ} \mathrm{C}\right) ; \mathrm{X} 2=$ Agitação $(\mathrm{rpm}) ; \mathrm{X} 3=\mathrm{pH} ; \mathrm{X} 4=$ Extrato de levedura $(\mathrm{g} / \mathrm{L}) ; \mathrm{X} 5=$ Extrato de malte $(\mathrm{g} / \mathrm{L}) ; \mathrm{X} 6=$ Peptona $(\mathrm{g} / \mathrm{L}) ; \mathrm{X} 7=\mathrm{Glicose}(\mathrm{g} / \mathrm{L})$ 
Conforme resultados apresentados na Tabela 2, a maior recuperação de carotenoides específicos $(697,24 \mu \mathrm{g} / \mathrm{g})$ foi obtida no ensaio 1, onde o meio de cultivo apresentava apenas glicose na sua composição, e as maiores faixas de temperatura $\left(30^{\circ} \mathrm{C}\right)$ e $\mathrm{pH}(6)$.

Silva (2009) testando o efeito do meio de cultura adicionado de efluente de parboilização de arroz, na otimização da produção de astaxantina por Phaffia rhodozyma (formalmente $X$. dedrorhous), obteve o melhor resultado $(538,4 \mu \mathrm{g} / \mathrm{g})$ utilizando $10 \mathrm{~g} / \mathrm{L}$ de extrato de levedura, $1 \mathrm{~g} / \mathrm{L}$ de extrato de malte, $10 \mathrm{~g} / \mathrm{L}$ de peptona, $5 \mathrm{~g} / \mathrm{L}$ de sacarose e $0 \mathrm{~g} / \mathrm{L}$ de efluente.

Valduga et al. (2008) utilizando resíduos agroindustriais para variar o meio de cultura na bioprodução de Sporidiobolus salmonicolor CBS 2636 e utilizando método de extração similar com DMSO, encontrou o maior resultado de carotenoides específicos $(137,8 \mu \mathrm{g} / \mathrm{g})$ empregando 17,1 g/L de melaço de cana, 1,4 g/L de água de maceração de milho e 1,4 g/L de levedura hidrolisada.

\section{CONCLUSÕES}

Neste trabalho foram investigadas as condições de bioprodução e variação no meio de cultura a fim de aumentar a produção e consequentemente a recuperação de carotenoides. Em meio composto apenas por $10 \mathrm{~g} / \mathrm{L}$ de glicose, com $150 \mathrm{rpm}$, temperatura de $30^{\circ} \mathrm{C}$ e pH 6 obtevese uma recuperação de $697,24 \mu \mathrm{g} / \mathrm{g}$ de carotenoides específicos, valores superiores aos encontrados na literatura.

\section{REFERÊNCIAS BIBLIOGRÁFICAS}

AKSU, Z.; EREN, A.T. Production of carotenoids by isolated yeast of Rhodotorula glutinis. Bioch.l Eng. Journal. v. 35, p. 107 - 113, 2007.

DA SILVA, M. C. Alteração na biossíntese de carotenoides em leveduras induzidas por agentes químicos. 2004. Tese (Doutorado em ciência de alimentos)- Universidade Estadual de Campinas, São Paulo, 2004.

JOHNSON, E. A.; SCHROEDER, W. A. Singlet oxygen and peroxyl radicals regulate carotenoid biosynthesis in Phaffia rhodozyma. Journal of Bio. Chem., v. 270, p. 18374 - 18379, 1995.

MACÍAS-SÁNCHEZ, M.D.; MANTELL, C.; RODRÍGUEZ, M.; MARTÍNEZ DE LA OSSA, E.; LUBIÁN, L. M.; MONTERO, O. Supercritical fluid extraction of carotenoids and clorophylla from Nannoclhoropsis gaditana. Journal of Food Eng., v. 66, p. 245-251, 2004.

MACÍAS-SÁNCHEZ, M. D.; FERNANDEZ-SEVILLA, J.M.; ACIÉN FERNANDEZ, F.G.; CERÓN GARCIA, M.C.; GRIMMA, E.M. Supercritical fluid extraction of carotenoids from Scenedesmus almeriensis. Food Chem.. V. 123, p. 928-935, 2010. 
MEZZOMO, N. Extração e encapsulamento de compostos com importância tecnológica e biológica proveniente do resíduo de processamento de camarão. Tese (Doutorado em Engenharia de Alimentos) - Universidade Federal de Santa Catarina, Santa Catarina, 2012.

MORIEL, D. G.; MACHADO, I. M. P.; FONTANA, J. D.; BONFIM, T. M. Optimization of biomass and astaxanthin production by the yeast Phaffia rhodozyma. Revista Brasileira de Ciências Farmacêuticas, Brazilian Journal of Pharm. Scie., V. 40, n. 3, jul./set., 2004.

RODRIGUES-AMAYA, D.B; KIMURA, M.; AMAYA-FARFAN, J. Fontes brasileiras de carotenoides: tabela brasileira de composição de carotenoides em alimentos. Brasília: MMA/SBF, 2008.

SILVA, D. A. Maximização da produção de astaxantina por Phaffia rhodozyma utilizando água de parboilização de arroz. Dissertação (Mestrado em Ciência e Tecnologia de Alimentos). Universidade Federal de Rio Grande. 2009.

VALDUGA, E. ; VALÉRIO, A.; TREICHEL, H.; DI LUCCIO, M.; FURIGO JUNIOR, A. Study of the bio-production of carotenoids by Sporidiobolus salmonicolor (CBS 2636) using pre-treated agro-industrial substrates. Journal of Chem. Tech. and Biotech., v.83, p.1267 - 1274, 2008.

VALDUGA, E.; TATSCH, P.O.; TIGGEMANN, L.; TREICHEL, H.; TONIAZZO, G.; ZENI, J.; DI LUCCIO, M. Produção de carotenoids: microrganismos como fonte de pigmentos naturais. Quím. Nova, v. 32, p. 2429-2436, 2009. 